\title{
FLASH
}

\section{TRANSPLANTATION DE NEURONES FOETAUX CHEZ DES PATIENTS PARKINSONIENS}

Deux transplantations de neurones humains embryonnaires ont été réalisées à l'hôpital Henri-Mondor de Créteil, au cours de l'année passée, chez des patients atteints de la maladie de Parkinson. Comme cela avait été le cas pour les malades opérés en Suède par l'équipe d'Olle Lindvall, les cellules provenant de la région mésencéphalique ventrale d'embryons de 6 à 9 semaines de gestation ont été mises en suspension puis implantées par voie stéréotaxique dans les noyaux gris centraux du côté le plus atteint. Une immunosuppression a été maintenue pendant les premiers mois puis allégée larrêt de la ciclosporine $A$ à 7 mois). Nous avons suivi scrupuleusement le protocole d'examen clinique et paraclinique (CAPIT) établi l'an dernier par un comité international afin de rendre nos résultats utilisables à terme pour un bilan de l'expérience. Les résultats obtenus chez les deux patients sont positifs puisque après, respectivement, 10 et 3 mois d'évolution, ils présentent une nette amélioration de tests de motricité du côté opposé à la transplantation. II ne s'agit cependant pas d'une transformation spectaculaire de la situation clinique et la dopa-thérapie doit être poursuivie. Elle est, apparemment, mieux tolérée après greffe neuronale. L'amélioration clinique apparaît dès la fin du premier mois et semble continuer de progresser 10 mois après l'intervention. La tomographie à émission de positons (réalisée en post-opératoire chez le premier patient pour l'instant) montre une augmentation de la capture du fluorodopa au site de la greffe, signant la présence de neurones dopaminergiques fonctionnels.

Ces premières transplantations intracérébrales en France, préparées depuis plusieurs années dans le cadre d'un réseau de recherche de l'Inserm, ne sont que le début d'une expérimentation thérapeutique. Celleci ne peut aller que lentement car chaque patient réclame un suivi clinique et paraclinique très lourd pour que l'effet de la transplantation puisse être jugée sur l'évolution de la maladie déjà traitée, en continu, par L-dopa. On est encore loin du passage éventuel de cette technique à la pratique courante. La transplantation neuronale apparaît toutefois comme une thérapeutique expérimentale prometteuse dans la maladie de Parkinson, qui peut se combiner avec la dopa-thérapie mais qui ne paraît pas devoir la remplacer.

Pierre Césaro Marc Peschanski

Réseau de Recherche Clinique de I'Inserm sur les Transplantations Intracérébrales 